\title{
ARTIGOS
}

Submetido 30.09.2019. Aprovado 01.04.2020

Avaliado pelo sistema double blind review. Editor Científico convidado: Edilson Paulo

Versão original

DOI: http://dx.doi.org/10.1590/S0034-759020200503

\section{CONCENTRAÇÃO ACIONÁRIA, CONSELHO DE ADMINISTRAÇÃO E REMUNERAÇÃO DE EXECUTIVOS}

\author{
Shareholder concentration, board structure and executive remuneration \\ Concentración accionaria, junta directiva y remuneración de ejecutivos
}

\section{MARIA RAFAELA DE OLIVEIRA FREITAS 1}

rafitadeut@gmail.com 0000-0002-4811-378X

GUSTAVO MAGNO PEREIRA ${ }^{2}$ gumagno@hotmail.com 0000-0001-9319-3451

\section{ALESSANDRA CARVALHO DE VASCONCELOS ${ }^{1}$} alevasconcelos.ufc@gmail.com 0000-0002-6480-5620

\section{MÁRCIA MARTINS MENDES DE LUCA ${ }^{1}$ \\ marciammdeluca@gmail.com \\ 0000-0002-9995-5637}

1Universidade Federal do Ceará, Programa de Pós Graduação em Administração e Controladoria, Fortaleza, CE, Brasil

2Universidade Federal Fluminense, Programa de Pós Graduação em Administração, Niterói, RJ, Brasil

\begin{abstract}
RESUMO
A investigação da influência da concentração acionária e da estrutura do conselho de administração no nível de remuneração de executivos permite esclarecer a dinâmica dos conflitos de agência presentes na empresa brasileira. Os resultados dessa investigação, em 232 empresas listadas na B3, no período 2014-2016, apontam que a concentração acionária influencia negativamente o nível de remuneração executiva, coerentemente com a Teoria da Agência. Ademais, características da estrutura do conselho de administração têm repercussão sobre a remuneração executiva, além do que algumas dessas características afetam a sensibilidade da remuneração à concentração acionária, que, contrariamente à Abordagem do Poder Gerencial, evidencia o predomínio do conflito principal-principal no Brasil.
\end{abstract}

PALAVRAS-CHAVE | Concentração acionária, conselho de administração, remuneração de executivos, conflitos de agência, poder gerencial.

\section{ABSTRACT}

Understanding the dynamics of agency conflicts in Brazilian firms requires investigating the influence of shareholder concentration and board structure in the remuneration level of executives. Based on a sample of 232 firms traded on B3 between 2014 and 2016, we found shareholder concentration to have a negative effect on executive remuneration, as predicted by the Agency Theory. The characteristics of the board structure also have repercussions on executive compensation. Some of these characteristics affect the sensitivity of compensation to shareholder concentration, which, contrary to the Managerial Power Approach., highlights the predominance of the principal-principal conflict in Brazil.

KEYWORDS I Shareholder concentration, board of directors, executive compensation, agency conflicts, managerial power.

\section{RESUMEN}

Investigar la influencia de la concentración accionaria y la estructura de la junta directiva en el nivel de remuneración de los ejecutivos arroja luz sobre la dinámica de los conflictos de agencia presentes en la empresa brasileña. Los resultados en 232 empresas que cotizan en la B3, en el período 2014-2016, apuntan a que la concentración accionaria influencia negativamente la remuneración ejecutiva, coherentemente con la Teoría de Agencia. Además, las características de la estructura de la junta directiva tienen repercusiones en la remuneración ejecutiva, y algunas de estas características afectan la sensibilidad de la remuneración a la concentración accionaria, lo que, al contrario de la Teoría del Poder Gerencial, destaca el predominio del conflicto principal-principal en Brasil.

PALABRAS CLAVE I Concentración accionaria, junta directiva, remuneración de ejecutivos, conflictos de agencia, poder de gerencial. 


\section{INTRODUÇÃO}

Principal órgão de governança corporativa, o conselho de administração (CA) desempenha essencial papel na definição da remuneração executiva, buscando a realização de contratos ótimos, que garantam alinhamento de interesses para maximizar retorno dos acionistas e reduzir custos de agência (Jensen, 1993).

Embora considerada custo de agência, a compensação de executivos é utilizada como importante mecanismo interno de governança, sendo empregada para alinhar interesses divergentes entre agente e principal (Jensen \& Meckling, 1976). Por outro lado, a alta gestão pode exercer influência sobre pacotes de compensação, porquanto a separação entre propriedade e controle oferece maior discricionariedade à gestão (Berle \& Means, 1932). Esse poder dos executivos de influenciar sua própria remuneração é conhecido como resultado da Abordagem do Poder Gerencial (Bebchuk \& Fried, 2003), decorrente do clássico conflito agente-principal, preconizado pela Teoria da Agência.

Em ambientes mais concentrados, cuja intensidade do conflito principal-principal, entre acionistas minoritários e controladores, passa a ser mais latente (Porta, Lopez-de-Silanes, \& Shleifer, 1999), os contratos de remuneração podem ser mecanismos menos relevantes de mitigação de conflitos entre agente e principal, haja vista que os próprios controladores exercem maior monitoramento sobre ações dos gestores, substituindo tarefa que caberia ao CA (Shleifer \& Vishny, 1997). Neste caso, a alta concentração acionária pode ser considerada um mecanismo substitutivo da remuneração executiva no alinhamento de interesses entre acionistas e gestores (Mehran, 1995).

Nesse contexto, a concentração acionária influencia o desenho dos pacotes de remuneração a executivos (Jiang, Habib, \& Smallman, 2009), segundo a estrutura proprietária e o ambiente institucional. Assim, há evidências de que, contrariamente às empresas com capital mais disperso, aquelas com alta concentração acionária, presentes em ambientes com fraca proteção legal a acionistas minoritários, tendem a remunerar seus executivos em níveis mais baixos (Hassen, Ouakdi, \& Omri, 2015).

No Brasil, mercado emergente caracterizado por alta concentração acionária, cujo maior acionista detém cerca de $59 \%$ do capital votante (Pinto \& Leal, 2013), é relevante ampliar a investigação, ainda incipiente na literatura nacional, acerca da influência da concentração sobre o nível de remuneração executiva, permitindo compreender conflitos de agência decorrentes dessa relação, que repercutem na empresa brasileira.

Ademais, conforme Core, Holthausen e Larcker (1999), a estrutura do CA, vista como mecanismo indicativo de qualidade da governança, também pode influenciar o nível de compensação executiva, na medida em que a alta gestão, exercendo influência sobre o CA, detém maior poder na definição da própria remuneração, conforme prevê a Abordagem do Poder Gerencial (Bebchuk \& Fried, 2003), o que agrava conflitos agente-principal.

No entanto, ao considerar o cenário de concentração de propriedade, em que o CA, muitas vezes, atua de maneira limitada devido ao poder de influência do controlador (Dutra \& Saito, 2002), atrelado ao baixo nível de qualidade de sua composição (Porta, López-de-Silanes, Shleifer, \& Vishny, 2000), é possível que a estrutura do CA venha interferir na relação entre concentração acionária e nível de remuneração.

Diante do exposto, sob o enfoque da Teoria da Agência (Jensen \& Meckling, 1976) e da Abordagem do Poder Gerencial (Bebchuk \& Fried, 2003), ainda pouco explorada no Brasil, este estudo tem o objetivo de investigar a influência da concentração acionária e da estrutura do CA sobre o nível de remuneração executiva de empresas brasileiras de capital aberto.

Para tanto, o estudo reúne 696 observações de 232 empresas não financeiras listadas na B3, no período 2014-2016. Os dados sobre remuneração executiva e estrutura do CA foram extraídos dos formulários de referência das empresas, já os dados financeiros e sobre concentração acionária foram coletados da base Economatica ${ }^{\circledR}$.

Os resultados sugerem que a concentração acionária reduz o nível de remuneração executiva, sinalizando que o monitoramento exercido pelo controlador parece substituir a remuneração de executivos na mitigação do conflito agenteprincipal (Hassen et al., 2015; Luo, 2015). Constatou-se, ainda, que a dualidade do CEO e a proporção de membros do CA atuantes na diretoria também reduzem o nível remuneratório, enquanto o percentual de membros independentes aumenta esse nivel.

Além disso, constatou-se que a proporção de membros internos, a de membros independentes e o tamanho do CA aumentam a sensibilidade da remuneração à concentração acionária, enquanto a proporção de membros indicados pelo controlador diminui essa sensibilidade, atestando o efeito moderador de aspectos da composição do CA. Tais resultados sugerem existir baixa repercussão da Abordagem do Poder Gerencial no Brasil, onde é predominante o conflito principalprincipal (Brandão \& Crisóstomo, 2015).

Destarte, esta pesquisa diferencia-se das anteriores (Correia, Amaral, \& Louvet, 2014; Ermel \& Monte, 2016; Pinto \& Leal, 2013) pela abordagem tanto da concentração acionária quanto da remuneração executiva e da estrutura do CA como mecanismos internos de governança que interagem entre si, permitindo tornar mais claro o desenho dos conflitos de agência no ambiente institucional brasileiro e ajudar as organizações nas decisões de melhoria de processos de governança. 


\section{REVISÃO DE LITERATURA E HIPÓTESES}

\section{Concentração acionária e remuneração executiva}

Existe evidência empírica de que a estrutura de propriedade, sob o aspecto da concentração acionária, interfere nos conflitos de agência, constatando-se que gestores têm maior poder discricionário em mercados com baixa concentração (Shleifer \& Vishny, 1997).

Em mercados com capital mais disperso, cuja separação entre propriedade e controle é predominante, em que gestores passam a ter maior discricionariedade para tomar decisões (Jensen \& Meckling, 1976), cabe ao CA o efetivo papel de estabelecer pacotes de compensação capazes de alinhar interesses entre acionistas e gestores (Bettis, Bizjak, Coles, \& Kalpathy, 2018).

Em contrapartida, a alta concentração acionária tende a mitigar os conflitos agente-principal, pois o controlador, pelo efeito substituição, assume o monitoramento sobre a gestão, que caberia ao CA (Dyck \& Zingales, 2004). No entanto, os controladores podem exacerbar benefícios privados de controle, apoiando-se num sistema de governança mais fraco (Bozec \& Bozec, 2007; Brandão \& Crisóstomo, 2015), o que leva à expropriação de acionistas minoritários, ampliando-se os conflitos principal-principal (Porta et al., 1999, 2000). Nesse cenário, a remuneração executiva tende a ser substituída, na mitigação dos conflitos agente-principal, pelo papel de monitoramento mais efetivo do controlador, o que justificaria níveis remuneratórios mais baixos (Almazan, Hartzell, \& Starks, 2005).

Young, Peng, Ahlstrom, Bruton e Jiang (2008) argumentam que a perspectiva de agente-principal pode ter aplicação limitada em economias emergentes, porque, nestas, a maioria das empresas tem controle familiar ou estatal, com propriedade concentrada. Isso resulta em maior potencial de conflitos entre majoritários e minoritários, predominante no mercado brasileiro de propriedade concentrada e de fraca proteção legal aos minoritários, que colabora para lento avanço da qualidade da governança (Brandão \& Crisóstomo, 2015; Pinto \& Leal, 2013).

$\mathrm{Na}$ literatura estrangeira, existem evidências que confirmam a influência da concentração acionária sobre o nível de remuneração executiva, que tende a níveis menos elevados em mercados de maior concentração (Croci, Gonenc, \& Ozkan, 2012; Firth, Fung, \& Rui, 2007; Hartzell \& Starks, 2003; Luo, 2015), contrariamente aos níveis de remuneração mais elevados em ambientes de maior dispersão acionária (Jiang et al., 2009; John, Mehran, \& Qian, 2010).

No Brasil, destacam-se pesquisas que convergem para a constatação de associação negativa, em que companhias com maior concentração pagam menor remuneração executiva (Anjos, Tavares, Monte, \& Lustosa, 2015; Ermel \& Monte, 2016; Pinto \& Leal 2013).

Com base no aporte teórico e empírico exposto, tendo em vista o cenário brasileiro de alta concentração acionária, espera-se existir influência negativa na remuneração executiva:

$\mathrm{H}_{1}$ : A maior concentração de direitos de voto dos principais acionistas associa-se negativamente ao nível de compensação de executivos.

Além de abordar a concentração acionária, a pesquisa avança na proposta de investigar a influência da estrutura do CA sobre a remuneração executiva, verificando, ainda, se a relação entre concentração e nível de remuneração é moderada por aspectos associados à estrutura do CA, sob o aporte teórico da Abordagem do Poder Gerencial, ainda pouco explorado no contexto brasileiro.

\section{Abordagem do Poder Gerencial e estrutura do conselho}

Em decorrência dos conflitos oriundos da Teoria da Agência, Bebchuk e Fried $(2003,2004)$ trazem uma abordagem teórica que torna mais expressivos os conflitos agente-principal, denominada Managerial Power Approach (Abordagem do Poder Gerencial). Segundo eles, com a separação entre propriedade e controle, a gestão passa a assumir maior discricionariedade nas decisões sobre os negócios, e isso pode levar à implementação de ações no melhor interesse da alta gestão, em detrimento do objetivo principal dos acionistas, de maximização do retorno de investimentos (Berle \& Means, 1932).

A Abordagem do Poder Gerencial considera que os acionistas não conseguem observar diretamente as ações dos gestores, que, por conhecerem mais sobre a empresa, são mais propensos ao comportamento oportunista com relação à própria remuneração, levando-os a extrair rendas excessivas, permitindo estabelecer vínculo entre poder gerencial e arranjos de pagamentos (Abascal \& González, 2019; Bebchuk \& Fried, 2004). Tal abordagem prevê que pacotes de compensação serão mais favoráveis aos gestores, principalmente, no caso em que o CA é relativamente fraco ou ineficaz. Segundo Bebchuk e Fried (2004), esse poder de influência da alta gestão sobre o CA colabora para intensificar os clássicos conflitos agente-principal.

Para a Abordagem do Poder Gerencial, grande parte dos estudos empíricos acerca da influência do poder dos gestores sobre a remuneração utiliza características da estrutura do CA 
como aproximação ao poder exercido pela alta gestão: tamanho; número de diretores executivos; e acumulação pelo CEO do cargo de presidente (Core et al., 1999; Newman \& Mozes, 1999). Ademais, este estudo acrescenta a proporção de membros do CA indicados pelo controlador e a de membros independentes, que tendem a refletir em redução desse poder gerencial e do nível remuneratório (Brandão, Vasconcelos, Luca, \& Crisóstomo, 2019; Choe, Tian, \& Yin, 2014).

Em contrapartida, existe evidência de que a concentração de propriedade pode ser prejudicial tanto à qualidade da governança quanto à composição do CA (Crisóstomo, Brandão, \& Lopéz-Iturriaga, 2020; Bozec \& Bozec, 2007; Porta et al., 2000), tendo em vista que grandes controladores podem utilizar benefícios privados de controle em detrimento de minoritários, conhecido por efeito expropriação decorrente do conflito principal-principal. Portanto, no contexto brasileiro de alta concentração acionária, uma vez que grandes controladores têm poder de influênciar a composição do órgão colegiado (Dutra \& Saito, 2002), é possível que aspectos atinentes à composição do CA venham influenciar a sensibilidade da remuneração à concentração acionária, interferindo nos conflitos de agência.

Segundo estudo seminal de Jensen (1993), o número de membros do CA é visto como fator importante para aumentar a eficácia desse colegiado no monitoramento da gestão. $\mathrm{Na}$ relação entre compensação executiva e tamanho do CA, estudos argumentam que, à medida que o tamanho do CA aumenta, o controle sobre a compensação executiva enfraquece, em decorrência de desentendimentos entre seus membros, da dificuldade de coordenação e redução de incentivos de monitoramento, facilitando a formulação de maior remuneração pelo CEO (Jensen, 1993; Lee \& Chen, 2011).

Ademais, existe evidência de que, em ambientes de alta concentração acionária, o tamanho do CA pode reduzir a complexidade de monitoramento das decisões dos gestores, e que um CA menor seria mais efetivo nesse monitoramento (Cheung, Stouraitis, \& Wong, 2005; Schiehll \& Santos, 2004).

A partir desses pressupostos, espera-se que executivos possam conseguir maior remuneração quando o CA é maior, reduzindo a sensibilidade da remuneração à concentração acionária:

H2: O maior número de membros do conselho associa-se positivamente ao nível de compensação executiva e reduz a sensibilidade da remuneração à concentração acionária.

Embora a separação entre funções de presidente do CA (chairman) e diretor presidente da empresa (CEO) seja considerada uma boa prática de governança corporativa, por garantir maior efetividade do monitoramento da gestão por aquele colegiado, ainda é comum a adoção de postura contrária pelas firmas. Contudo,
Conyon e Peck (1997) destacam a importância da separação de funções para reduzir o poder discricionário gerencial.

Finkelstein (1992), por sua vez, assevera que o principal canal utilizado pelo CEO para ampliar seu poder é o CA, especialmente quando assume também o posto de chairman, o que amplia seu poder político no contexto da empresa, reforçando comportamentos gerenciais oportunísticos. Assim, seguindo evidência empírica, a remuneração executiva será maior em firmas com controle mais fraco sobre o CEO, em que existe dualidade de funções, pois invalida controles efetivos do CA sobre o diretor presidente (Bugeja, Rosa, Duong, \& Izan, 2012; Lin \& Lu, 2009).

Cabe destacar, ainda, que a dualidade do CEO parece estar mais presente em firmas com grandes acionistas majoritários, sugerindo maior facilidade de concentração de poder nas mãos de poucos controladores, comprometendo a habilidade do CA de monitorar de modo independente e eficaz (Cheung et al., 2005; Schiehll \& Santos, 2004):

H3: A dualidade do CEO associa-se positivamente ao nível de compensação executiva e reduz a sensibilidade da remuneração à concentração acionária.

Conforme estudos prévios, um CA caracterizado pela participação de grande proporção de diretores internos pode dificultar a eficácia de sua função de monitoramento, pois os diretores internos tendem a concordar com o CEO na definição de estratégias que se distanciam do interesse dos acionistas (Grabke-Rundell \& Gomez-Mejia, 2002).

Assim, grande proporção de diretores internos no CA ofereceria aos executivos oportunidade adicional para se favorecerem com propostas remuneratórias que lhes seriam mais vantajosas, em detrimento do menor esforço desses executivos de perseguir estratégias maximizadoras da performance da firma (Bugeja et al., 2012).

Vale ressaltar que a literatura sugere ser mais comum, em ambiente de alta concentração acionária, a participação de insiders, diretores internos, no CA, do que a de outsiders, comprometendo esforços de monitoramento do conselho (Cheung et al., 2005; Schiehll \& Santos, 2004).

Dessa forma, investiga-se, nas empresas brasileiras, a influência da proporção de diretores internos no CA sobre o nível de compensação executiva e sua sensibilidade à concentração acionária:

H4: A maior proporção de diretores executivos no conselho associa-se positivamente ao nível de compensação executiva e reduz a sensibilidade da remuneração à concentração acionária. 
Segundo Core et al. (1999), o CA deve atuar como órgão de controle em nome dos acionistas, e, para desempenhá-lo efetivamente, a independência do órgão torna-se essencial, sendo garantida quando seus membros externos não mantêm qualquer afiliação com a firma (Instituto Brasileiro de Governança Corporativa - IBGC, 2015). Desse modo, alguns estudos propuseram associação negativa entre independência do CA e nível de remuneração do CEO, argumentando que grande número de membros independentes sinaliza redução do poder do CEO para obter vantagens remuneratórias (Ozdemir \& Upneja, 2012).

Segundo Brandão et al. (2019), essa independência tornaria possível mitigar conflitos de agência tanto entre acionistas e gestores quanto entre minoritários e majoritários. Assim, a independência do CA pode reduzir o poder dos executivos de influenciar as deliberações do órgão sobre sua remuneração, evitando-se extração excessiva de rendas remuneratórias (Chhaochharia \& Grinstein, 2009).

No entanto, a independência do CA pode ser prejudicada em firmas de propriedade concentrada, uma vez que grandes controladores tendem a participar ou até indicar membros familiares para compor o CA (Cheung et al., 2005; Schiehll \& Santos, 2004). Do exposto, espera-se existir influência negativa da proporção de membros independentes sobre o nível de remuneração executiva e consequente aumento da sensibilidade à concentração:

$\mathrm{H}_{5}$ : A maior proporção de membros independentes no conselho associa-se negativamente ao nível de compensação executiva e aumenta a sensibilidade da remuneração à concentração acionária.

No contexto de mercados com propriedade mais concentrada, como no Brasil, é comum o CA ser constituído por membros indicados pelo controlador (Dutra \& Saito, 2002), o que poderia diminuir a influência dos executivos sobre a própria remuneração, tendo em vista o monitoramento exercido pelo controlador e pelos membros do CA por ele indicados (Claessens \& Yurtoglu, 2013). Entretanto, nesse cenário, embora os conflitos agente-principal sejam mitigados, os conflitos principal-principal são acentuados (Brandão \& Crisóstomo, 2015; Chen \& Keefe, 2018). Além disso, Bertucci, Bernardes e Brandão (2006) defendem que os majoritários são influenciadores das decisões relativas à remuneração executiva. Dessa forma, propõe-se:

H6: A maior proporção de membros do conselho indicados pelo acionista controlador associa-se negativamente ao nível de compensação executiva e aumenta a sensibilidade da remuneração à concentração acionária.

\section{METODOLOGIA}

A população reúne todas as empresas de capital aberto listadas na B3, optando-se pelo período 2014-2016, cujos dados estiveram disponíveis na data da realização da pesquisa. Dessa população, são analisadas as empresas não financeiras que divulgaram os dados necessários ao longo do período de análise, totalizando uma amostra de 232 firmas e 696 observações.

Os dados sobre remuneração executiva, assim como aqueles referentes à estrutura do CA, foram extraídos do formulário de referência de cada empresa, disponível no website da Comissão de Valores Mobiliários (CVM), enquanto os dados financeiros e de concentração acionária foram coletados da base Economática ${ }^{\circledR}$, no período de setembro a novembro de 2017.

O modelo empírico utilizado é o de regressão linear múltipla, estimado pelo Método dos Mínimos Quadrados Ordinários (MQO), sendo o modelo mais adequado e disseminado em pesquisas sobre determinantes da remuneração executiva (Anjos et al., 2015; Ermel \& Monte, 2016; Pinto \& Leal, 2013; Theiss \& Beuren, 2017). As variáveis independentes são representadas pela concentração acionária e estrutura do CA, cujos efeitos sobre a variável dependente, remuneração executiva, são analisados considerando a interação entre elas. Foram utilizadas, ainda, variáveis de controle mais recorrentes nos estudos sobre o tema: tamanho, desempenho e endividamento da firma. Destarte, a Equação 1 traz a proposição do modelo empírico do estudo.

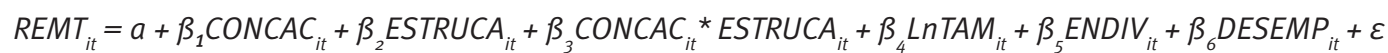

Em que:

$R E M T_{i t}$ é a remuneração total da diretoria executiva da empresa $i$ no período $t$;

CONCAC $_{i t}$ representa as variáveis indicativas da concentração acionária, em termos de direitos de voto dos principais acionistas, da empresa $i$ no período $t$; 
ESTRUCA $_{i t}$ representa as variáveis indicativas da estrutura do CA da empresa $i$ no período $t$;

$\operatorname{LnTAM}_{i t}$ representa o tamanho da empresa $i$ no período $t$

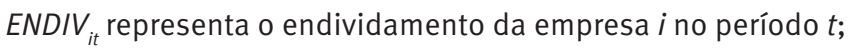

$D E S E M P_{i t}$ representa o desempenho da empresa $i$ no período $t$;

$a$ é o intercepto da reta;

$\beta_{1}, \beta_{2}, \beta_{3}, \beta_{4}, \beta_{5}$ e $\beta_{6}$ são os coeficientes angulares das variáveis explicativas;

$\varepsilon$ é o termo de erro da equação.

Vale ressaltar que $\operatorname{CONCAC}_{i t}$ * $E S T R U C A_{i t}$ representa variável de interação cujo coeficiente $\left(\beta_{3}\right)$ testa se as variáveis indicativas da estrutura do CA afetam a sensibilidade da remuneração à concentração acionária $\left(\beta_{1}\right)$. Ademais, estimações adicionais, não reportadas, foram realizadas, incluindo variáveis de controle (ano da observação e setor de atividade), porém seus coeficientes não apresentaram significância estatística e não alteraram a significância das variáveis de interesse.

O Quadro 1 traz o detalhamento da operacionalização das variáveis do estudo.

\section{Quadro 1. Descrição das variáveis}

\begin{tabular}{|c|c|}
\hline Nível de remuneração da diretoria executiva (REMT) & Logaritmo natural (Ln) do total da remuneração da diretoria executiva (LnREMT) \\
\hline Concentração acionária (CONCAC) & $\begin{array}{l}\text { Proporção de ações ordinárias pertencentes ao maior acionista }\left(\mathrm{CVT}_{1}\right) \text {; dois maiores } \\
\left(\mathrm{CVT}_{2}\right) \text {; três maiores }\left(\mathrm{CVT}_{3}\right) \text {; quatro maiores }\left(\mathrm{CVT}_{4}\right) \text {; e cinco maiores acionistas }\left(\mathrm{CVT}_{5}\right)\end{array}$ \\
\hline Dualidade do CEO (DUALCA) & $\begin{array}{l}\text { Acúmulo dos cargos de CEO e chairman (variável dummy: o quando não existe } \\
\text { acúmulo de cargos; } 1 \text { quando existe acúmulo de cargos) }\end{array}$ \\
\hline Membros do CA integrantes da diretoria dxecutiva (DIRCA) & Proporção de membros titulares do CA que ocupam cargo de diretoria \\
\hline Membros do CA indicados pelo controlador (CONTCA) & Proporção de membros titulares do CA indicados pelo controlador \\
\hline Tamanho da empresa (LnTAM) & Logaritmo natural (Ln) do valor do Ativo \\
\hline Endividamento da empresa (ENDV) & Razão entre o valor da dívida total e o do Ativo \\
\hline Desempenho da empresa (DESEM) & Razão entre o valor do lucro líquido e o do Ativo \\
\hline
\end{tabular}

Destaca-se que as variáveis de composição da estrutura do CA são calculadas em relação ao total de membros efetivos do CA de cada firma. 


\section{RESULTADOS}

\section{Estatística descritiva}

A Tabela 1 apresenta as estatísticas descritivas das variáveis do modelo. A remuneração média bruta anual da Diretoria Executiva das empresas alcança cerca de $R$ \$10,2 milhões, com baixa variabilidade na amostra, segundo coeficiente de variação. Esses valores estão próximos aos encontrados por Anjos et al. (2015) em firmas da BM\&FBovespa no período 2011-2013.

\section{Tabela 1. Estatística descritiva das variáveis}

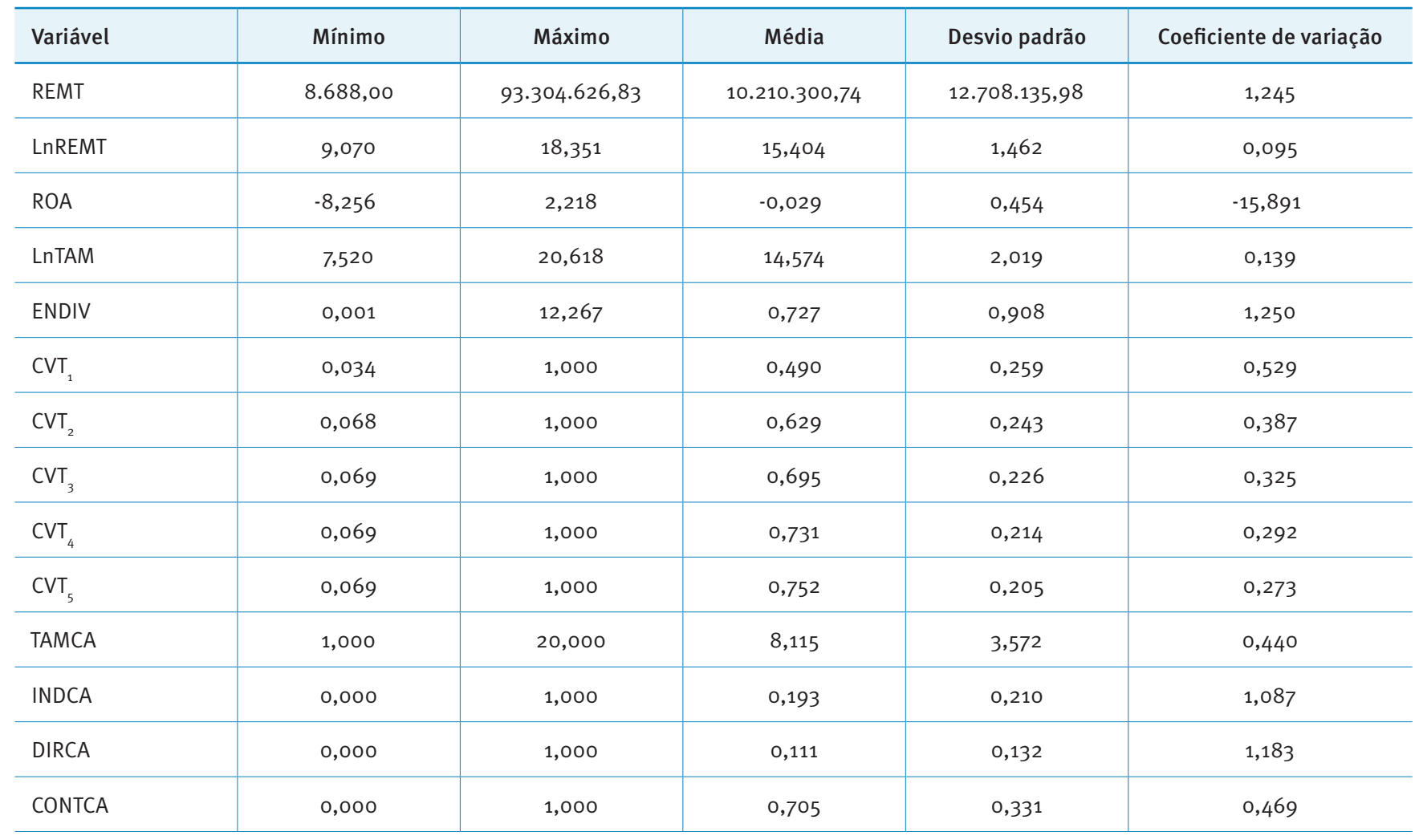

Nota: REMT = nível de remuneração da diretoria executiva; LnREMT = logaritmo natural do total da remuneração da diretoria executiva; ROA = retorno sobre o ativo; LnTAM = logaritmo natural do valor do ativo; ENDIV = endividamento; $\mathrm{CVT}_{1}=$ proporção de ações ordinárias pertencentes ao maior acionista; $C V T_{2}=$ proporção de ações ordinárias pertencentes aos dois maiores acionistas; $\mathrm{CVT}_{3}=$ proporção de ações ordinárias pertencentes aos três maiores acionistas; $\mathrm{CVT}_{4}=$ proporção de ações ordinárias pertencentes aos quatro maiores acionistas; $C V T$ = proporção de ações ordinárias pertencentes aos cinco maiores acionistas; TAMCA = tamanho do CA; INDCA = membros independentes do CA; DIRCA = membros titulares do CA que ocupam cargo de diretoria; CONTCA = membros do CA indicados pelo controlador.

Com relação à concentração acionária, registra-se proporção média de $49 \%$ nas mãos do principal acionista, crescendo até chegar a 75,2\% pertencentes aos cinco maiores acionistas, com baixa variabilidade na amostra, evidenciando elevados índices de concentração de direitos de voto, o que corrobora estudos prévios no Brasil, confirmando que empresas brasileiras assinalam elevada concentração (Brandão \& Crisóstomo, 2015; Silveira, Leal, Carvalhal-da-Silva, \& Barros, 2010).

Quanto à estrutura de composição do conselho, as empresas registram média de oito membros por CA, com baixa variabilidade dos dados, resultado que se aproxima das recomendações do IBGC (2015), assim como identificado por Brandão et al. (2019), em firmas do IBRX 100, no período 20132015 .

Além disso, em média 19,3\% dos membros são declarados independentes, enquanto $11,1 \%$ dos membros ocupam cargo de diretoria. Por outro lado, é alta a proporção média de membros indicados pelo controlador, que se aproxima dos 70\%. Esses números são consistentes com os encontrados por Brandão e Crisóstomo (2015) nas maiores companhias abertas brasileiras, no período 2010-2013.

Os resultados da correlação entre as variáveis quantitativas são apresentados na Tabela 2. 
Tabela 2. Correlação entre variáveis métricas

\begin{tabular}{|c|c|c|c|c|c|c|c|c|c|c|c|c|c|}
\hline VARIÁVEL & LNREMT & TAMCA & INDCA & DIRCA & CONTCA & LNTAM & ENDIV & ROA & $\mathrm{CVT}_{1}$ & $\mathrm{CVT}_{2}$ & $\mathrm{CVT}_{3}$ & $\mathrm{CVT}_{4}$ & $\mathrm{CVT}_{5}$ \\
\hline LNREMT & 1,000 & & & & & & & & & & & & \\
\hline TAMCA & $0,441^{(\star \star)}$ & 1,000 & & & & & & & & & & & \\
\hline INDCA & $0,290^{(* *)}$ & 0,025 & 1,000 & & & & & & & & & & \\
\hline CONTCA & $-0,125^{(\star \star)}$ & $-0,065$ & $-0,446^{(\star \star)}$ & $0,110^{(\star \star)}$ & 1,000 & & & & & & & & \\
\hline LNTAM & $0,717^{(\star \star)}$ & $0,597^{(* *)}$ & $0,196^{(\star *)}$ & $-0,362^{(* \star)}$ & $-0,034$ & 1,000 & & & & & & & \\
\hline $\mathrm{CVT}_{1}$ & $-0,259^{(\star \star)}$ & $-0,055$ & $-0,422^{(* \star)}$ & $0,088^{(*)}$ & $0,356^{(\star *)}$ & $-0,132^{(\star \star)}$ & $0,144^{(\star *)}$ & $-0,075^{(*)}$ & 1,000 & & & & \\
\hline $\mathrm{CVT}_{2}$ & $-0,334^{(\star *)}$ & $-0,069$ & $-0,471^{(\star \star)}$ & $0,117^{(\star \star)}$ & $0,357^{(* *)}$ & $-0,194^{(* *)}$ & $0,135^{(\star *)}$ & $-0,067$ & $0,909^{(* *)}$ & 1,000 & & & \\
\hline $\mathrm{CVT}_{3}$ & $-0,370^{(* \star)}$ & $-0,089^{(*)}$ & $-0,484^{(* *)}$ & $0,156^{(\star *)}$ & $0,338^{(* *)}$ & $-0,224^{(\star \star)}$ & $0,155^{(\star \star)}$ & $-0,063$ & $0,826^{(\star *)}$ & $0,967^{(\star \star)}$ & 1,000 & & \\
\hline $\mathrm{CVT}_{4}$ & $-0,377^{(* *)}$ & $-0,101^{(\star *)}$ & $-0,493^{(\star *)}$ & $0,153^{(* \star)}$ & $0,331^{(\star *)}$ & $-0,234^{(* *)}$ & $0,137^{(\star *)}$ & $-0,053$ & $0,750^{(\star \star)}$ & $0,913^{(\star \star)}$ & $0,981^{(\star \star)}$ & 1,000 & \\
\hline $\mathrm{CVT}_{5}$ & $-0,386^{(\star *)}$ & $-0,120^{(* *)}$ & $-0,491^{(\star)}$ & $0,159^{(\star \star)}$ & $0,318^{(* *)}$ & $-0,253^{(\star \star)}$ & $0,126^{(\star *)}$ & $-0,046$ & $0,700^{(\star *)}$ & $0,870^{(\star \star)}$ & $0,952^{(* *)}$ & $0,990^{(\star \star)}$ & 1,000 \\
\hline
\end{tabular}

Nota: ${ }^{(*),(* *),(* *)}$ denotam a significância estatística dos coeficientes em níveis de $10 \%, 5 \%$ e 1\%, respectivamente.

LnREMT = logaritmo natural do total da remuneração da diretoria executiva; TAMCA = tamanho do CA; INDCA = membros independentes do CA; DIRCA = membros titulares do CA que ocupam cargo de diretoria; CONTCA = membros do CA indicados pelo controlador; LnTAM = logaritmo natural do valor do ativo; ENDIV = endividamento; ROA = retorno sobre o ativo; $C V T$ = proporção de ações ordinárias pertencentes ao maior acionista; CVT = proporção de ações ordinárias pertencentes

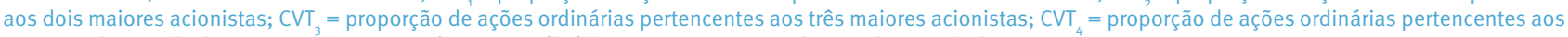
quatro maiores acionistas; $\mathrm{CVT}_{5}=$ proporção de ações ordinárias pertencentes aos cinco maiores acionistas.

Observa-se correlação negativa entre concentração acionária e nível de remuneração executiva, assim como encontrado em outros mercados emergentes (Firth et al., 2007; Luo, 2015). Em relação às variáveis da estrutura do CA, nota-se que o tamanho e a proporção de membros independentes estão correlacionados positivamente com o nível de remuneração executiva. Ademais, correlações negativas são identificadas tanto em relação à proporção de diretores membros do CA quanto em relação à de membros indicados pelo controlador.

Ao considerar a interação entre concentração acionária e variáveis da estrutura do CA, nota-se que existe correlação negativa com tamanho e independência do CA, e positiva com proporção de diretores internos e de membros indicados pelo controlador, apontando que a alta concentração pode estar contribuindo para a baixa qualidade da governança e, consequentemente, da composição do CA (Crisóstomo et al., 2020).

\section{Análise de regressão}

A Tabela 3 exibe os principais resultados descritos a partir das 25 estimativas da equação do modelo de regressão que verificam a influência da concentração acionária e da estrutura do CA sobre o nível de remuneração executiva. Destaca-se que, para todas as estimações do modelo, foi evidenciada ausência de multicolinearidade entre as variáveis explicativas, atestada pelos baixos valores (entre 1,00 e 1,62) dos respectivos Variance Inflation Factors (VIF) (Fávero, Belfiore, Silva, \& Chan, 2009). Outrossim, a independência dos resíduos, por meio do teste de Durbin-Watson, foi constatada por valores $p$ elevados para todas as estimações. Os testes de heterocedasticidade de Breusch-Pagan/Cook-Weisberg aplicados apontaram problemas de heterocedasticidade, tratados por meio da adoção de erros padrão robustos, obtidos pela correção robusta de White, em todas as análises. 
ARTIGOS | CONCENTRAÇÃO ACIONÁRIA, CONSELHO DE ADMINISTRAÇÃO E REMUNERAÇÃO DE EXECUTIVOS

Maria Rafaela de Oliveira Freitas | Gustavo Magno Pereira | Alessandra Carvalho de Vasconcelos | Márcia Martins Mendes De Luca

Tabela 3. Resultados para o modelo da Equação 1

\begin{tabular}{|c|c|c|c|c|c|c|c|c|c|c|}
\hline \multirow{2}{*}{$\begin{array}{l}\begin{array}{l}\text { Variável } \\
\text { Independente }\end{array} \\
\mathrm{CVT}_{1}\end{array}$} & \multicolumn{2}{|c|}{ (i) } & \multicolumn{2}{|c|}{ (ii) } & \multicolumn{2}{|c|}{ (iii) } & \multicolumn{2}{|c|}{ (iv) } & \multicolumn{2}{|c|}{ (v) } \\
\hline & $-0,913$ & $(\star \star \star)$ & $-0,674$ & $(* \star *)$ & $-0,899$ & $(* \star \star)$ & $-0,800$ & $(* \star *)$ & $-0,850$ & $\left(^{(\star \star \star)}\right.$ \\
\hline INDCA & & & 0,718 & $(* \star *)$ & & & & & & \\
\hline DIRCA & & & & & $-1,581$ & $(* \star \star)$ & & & & \\
\hline CONTCA & & & & & & & $-0,231$ & $(*)$ & & \\
\hline $\mathrm{CVT}_{1}$ * INDCA & & & $-3,299$ & $(* \star \star)$ & & & & & & \\
\hline $\mathrm{CVT}_{1}^{*}$ DIRCA & & & & & 1,802 & & & & & \\
\hline $\mathrm{CVT}_{1}{ }^{*} \mathrm{CONTCA}$ & & & & & & & 2,269 & $(* \star *)$ & & \\
\hline $\mathrm{CVT}_{1}{ }^{*}$ DUALCA & & & & & & & & & 0,513 & \\
\hline ROA & $-0,067$ & & $-0,059$ & & $-0,040$ & & $-0,077$ & & $-0,039$ & \\
\hline $\mathrm{N}$ & 696 & & 696 & & 696 & & 696 & & 696 & \\
\hline R2 & 0,545 & & 0,553 & & 0,561 & & 0,547 & & 0,549 & \\
\hline $\mathrm{F}$ & 165,33 & & 170,85 & & 176,39 & & 166,66 & & 168,59 & \\
\hline Valor $p$ & 0,000 & & 0,000 & & 0,000 & & 0,000 & & 0,000 & \\
\hline $\mathrm{CVT}_{2}$ & $-1,197$ & $(* \star *)$ & $-0,973$ & $(\star \star \star)$ & $-1,167$ & $(* \star \star)$ & $-1,103$ & $(* \star *)$ & $-1,121$ & $(* \star *)$ \\
\hline TAMCA & 0,013 & & & & & & & & & \\
\hline INDCA & & & 0,553 & $(* * *)$ & & & & & & \\
\hline DIRCA & & & & & $-1,544$ & $(\star \star \star)$ & & & & \\
\hline CONTCA & & & & & & & $-0,166$ & & & \\
\hline DUALCA & & & & & & & & & $-0,312$ & $(* *)$ \\
\hline $\mathrm{CVT}_{2}{ }^{*} \mathrm{TAMCA}$ & $-0,080$ & $(*)$ & & & & & & & & \\
\hline ENDIV & $-0,094$ & $(* \star)$ & $-0,093$ & $\left({ }^{* \star}\right)$ & $-0,033$ & & $-0,100$ & $(* \star)$ & $-0,073$ & \\
\hline Constante & 9,257 & $(* \star *)$ & 8,998 & $(\star \star \star)$ & 9,718 & $(\star \star \star)$ & 9,204 & $(* \star *)$ & 9,217 & ${ }^{(\star \star *)}$ \\
\hline $\mathrm{N}$ & 696 & & 696 & & 696 & & 696 & & 696 & \\
\hline R2 & 0,557 & & 0,561 & & 0,572 & & 0,558 & & 0,560 & \\
\hline $\mathrm{F}$ & 173,86 & & 176,80 & & 184,75 & & 174,22 & & 176,07 & \\
\hline Valor $p$ & 0,000 & & 0,000 & & 0,000 & & 0,000 & & 0,000 & \\
\hline $\mathrm{CVT}_{3}$ & $-1,404$ & $(* \star *)$ & $-1,188$ & $(* \star *)$ & $-1,351$ & $(\star \star \star)$ & $-1,314$ & $(* \star *)$ & $-1,323$ & $(\star \star \star)$ \\
\hline TAMCA & 0,013 & & & & & & & & & \\
\hline INDCA & & & 0,478 & $(* \star)$ & & & & & & \\
\hline DIRCA & & & & & $-1,470$ & $(* \star *)$ & & & & \\
\hline CONTCA & & & & & & & $-0,153$ & & & \\
\hline DUALCA & & & & & & & & & $-0,283$ & $(* *)$ \\
\hline $\mathrm{CVT}_{3}{ }^{*} \mathrm{TAMCA}$ & $-0,016$ & & & & & & & & & \\
\hline $\mathrm{CVT}_{3}{ }^{*}$ INDCA & & & $-1,246$ & & & & & & & \\
\hline $\mathrm{CVT}_{3}{ }^{*}$ DIRCA & & & & & $-2,997$ & $(* *)$ & & & & \\
\hline $\mathrm{CVT}_{3}{ }^{*} \mathrm{CONTCA}$ & & & & & & & 1,628 & $(* \star *)$ & & \\
\hline $\mathrm{CVT}_{3}$ * DUALCA & & & & & & & & & 0,114 & \\
\hline ROA & $-0,038$ & & $-0,037$ & & $-0,014$ & & $-0,046$ & & $-0,017$ & \\
\hline
\end{tabular}


Tabela 3. Resultados para o modelo da Equação 1

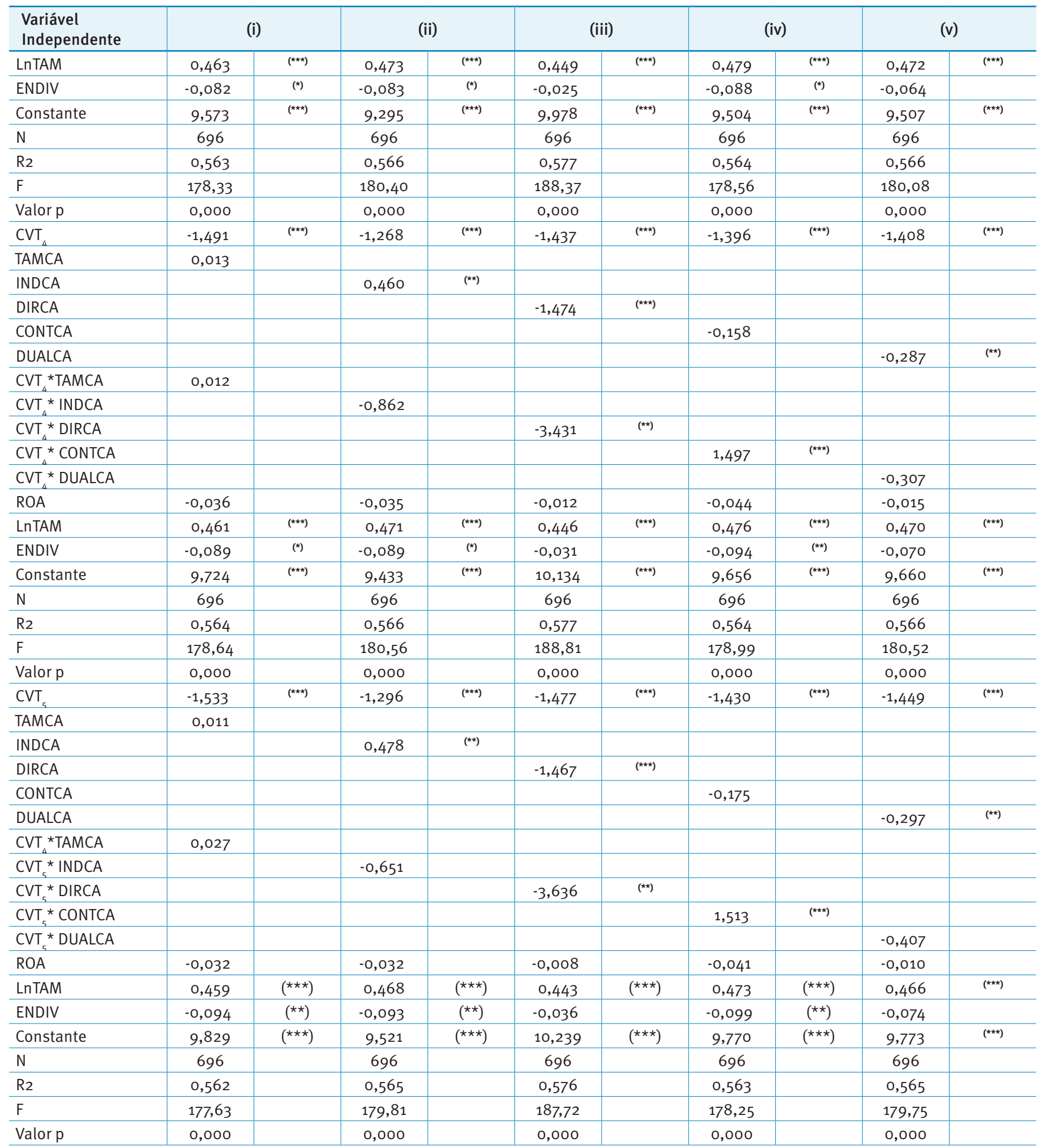

Nota: ${ }^{(*)},(* *),(* *)$ denotam a significância estatística dos coeficientes em níveis de $10 \%, 5 \%$ e 1\%, respectivamente. (i), (ii), (iii), (iv) e (v) representam estimações do modelo.

TAMCA = tamanho do CA; INDCA = membros independentes do CA; DIRCA = membros titulares do CA que ocupam cargo de diretoria; CONTCA = membros do CA indicados pelo controlador; DUALCA = dualidade do CEO; LnTAM = logaritmo natural do valor do ativo; ENDIV = endividamento; ROA = retorno sobre o ativo; CVT = proporção de ações ordinárias pertencentes ao maior acionista; $\mathrm{CVT}_{2}=$ proporção de ações ordinárias pertencentes aos dois maiores acionistas; $\mathrm{CVT}_{3}=$ proporção de ações ordinárias pertencentes aos três maiores acionistas; $\mathrm{CVT}_{4}=$ proporção de ações ordinárias pertencentes aos quatro maiores acionistas; $\mathrm{CVT}_{5}=$ proporção de ações ordinárias pertencentes aos cinco maiores acionistas. 
Confirma-se, em nível de significância de $1 \%$, a primeira hipótese $\left(\mathrm{H}_{1}\right)$, de que a concentração de ações ordinárias influencia negativamente o nível de remuneração executiva. Esse resultado é consistente com modelos estimados segundo diferentes medidas de concentração, que compreende a soma das ações ordinárias nas mãos dos cinco principais acionistas. Tal constatação alinha-se a achados em diversos mercados, que confirmam associação negativa da concentração acionária com compensação executiva (Hassen et al., 2015; Luo, 2015).

Os resultados das estimações indicam que, isoladamente, o tamanho do CA não exerce qualquer influência sobre o nível de remuneração, no entanto, ao interagir com a concentração acionária, aumenta a sensibilidade da relação entre concentração e remuneração executiva na presença do maior e dois maiores acionistas, levando à rejeição da segunda hipótese $\left(\mathrm{H}_{2}\right)$.

Com relação à influência da dualidade do CEO sobre a remuneração executiva, observa-se associação negativa, contrária ao esperado, para a terceira hipótese $\left(\mathrm{H}_{3}\right)$. No entanto, ao interagir com a variável concentração acionária, não se verifica qualquer influência da dualidade na sensibilidade da remuneração à concentração. Contrariamente ao prognosticado pela quarta hipótese $\left(\mathrm{H}_{4}\right)$, notou-se associação negativa entre membros internos e a remuneração, além do que, na interação com a concentração, notou-se que a proporção de membos internos aumenta a sensibilidade da remuneração à concentração, na presença dos três até os cinco maiores acionistas.

Apurou-se, ainda, que a proporção de membros independentes influencia positivamente o nível de remuneração executiva, contrariando a quinta hipótese $\left(\mathrm{H}_{5}\right)$, que previa relação inversa. Entretanto, ao considerar a interação com a variável concentração acionária, constatou-se que a independência do CA tende a aumentar a sensibilidade da remuneração à concentração, na presença do primeiro até os dois maiores acionistas.

Por outro lado, a proporção de membros indicados pelo controlador, isoladamente, não apresentou influência negativa significante sobre o nível de remuneração executiva, conforme proposição da sexta hipótese (H6); no entanto, em todos os modelos estimados, ao interagir com a concentração acionária, aquela diminui a sensibilidade da remuneração à concentração. Vale notar ainda, relativamente às variáveis de controle, que o tamanho da firma associa-se positivamente à remuneração executiva, evidenciando que empresas maiores pagam mais a seus executivos (Cao, Pan, \& Tian, 2011).

No geral, quanto à estrutura do CA, observa-se divergência de resultados em relação a achados prévios da literatura estrangeira, o que sinaliza, no Brasil, direção oposta à Abordagem do Poder Gerencial (Bebchuk \& Fried, 2003, 2004).

\section{DISCUSSÃO}

Constata-se que a influência negativa exposta pelas métricas de concentração acionária sobre o nível de remuneração executiva é consistente com o argumento de que controladores podem não ver a política de remuneração executiva como relevante, e tendem a remunerar a alta gestão a níveis mais baixos, sinalizando o efeito substituição da remuneração pelo monitoramento do controlador na mitigação de conflitos agente-principal (Almazan et al., 2005).

Essa associação negativa entre concentração acionária e nível de remuneração executiva, aqui encontrada, está de acordo com estudos no Brasil (Anjos et al., 2015; Ermel \& Monte, 2016; Pinto \& Leal, 2013) e em cenários estrangeiros, como Ásia, EUA e Europa (Croci et al., 2012; Firth et al., 2007; Hartzell \& Starks, 2003; Hassen et al., 2015; Luo, 2015).

Tais resultados devem-se ao fato de que elevada concentração acionária produz efeitos sobre relações de agência. Segundo Dyck e Zingales (2004), o reduzido número de controladores, detentores de maior poder, apresenta incentivos para se favorecer de benefícios privados de controle, levando-os a desconsiderar o custo com níveis mais elevados de remuneração executiva (Cao et al., 2011; John et al., 2010). Por outro lado, embora contribua para minimizar os conflitos agente-principal, o poder exercido pelo controlador superestima conflitos com minoritários (Luo, 2015).

Resultados contrários ao esperado para membros internos e dualidade do CEO, que apontam significância negativa para o nível de remuneração executiva, sinalizam que a Abordagem do Poder Gerencial não apresenta repercussão no Brasil, o que pode ser explicado por ser o conflito principal-principal o tipo mais acentuado no ambiente das empresas brasileiras de propriedade concentrada e familiar, cujo poder do controlador tende a interferir na escolha dos principais executivos (Brandão \& Crisóstomo, 2015; Pinto \& Leal, 2013), o que corrobora a redução do poder dos gestores neste cenário específico, justificando a propensão dessas empresas a aplicar menores níveis remuneratórios.

Quando se considera sua interação com a concentração acionária, nota-se que tanto a proporção de membros internos quanto o tamanho do CA aumentam a sensibilidade da remuneração à concentração, o que pode ser explicado pela baixa qualidade da composição do CA em ambiente de alta concentração acionária (Crisóstomo et al., 2020), cuja presença de controlador, por efeito substituição, compromete a eficácia de monitoramento do CA. Tal fato pode exercer influência sobre os membros do CA para definição de políticas de interesse do controlador, como menores níveis de remuneração executiva, a 
fim de garantir-lhe benefícios privados de controle, em detrimento dos minoritários, conduzindo ao efeito expropriação.

Com relação à influência positiva da proporção de membros independentes sobre o nível de remuneração executiva, uma possível explicação para tal resultado pode estar no trade off entre monitoramento e remuneração, evidenciando que a independência do CA considera relevantes os incentivos financeiros para alinhar interesses tanto entre gestores e acionistas quanto entre minoritários e majoritários (Mehran, 1995).

No entanto, ao interagir com a concentração acionária, constata-se que a proporção de membros independentes aumenta a sensibilidade da remuneração à concentração, o que pode ser explicado pelo fato de a independência do CA, embora seja indicativo de qualidade de governança, ser pouco valorizada no ambiente de alta concentração, maximizando o conflito principal-principal. Quanto à proporção de membros indicados pelo controlador, que reduz a sensibilidade à concentração, uma possível explicação estaria no fato de que grandes controladores tendem a participar ou até mesmo indicar membros familiares para compor o CA - prática conhecida como tunneling (Cheung et al., 2005) -, facilitando a extração direta de benefícios privados de controle por meio de níveis remuneratórios elevados.

\section{CONCLUSÃO}

Sob os fundamentos da Teoria da Agência e da Abordagem do Poder Gerencial, esta pesquisa investigou a influência da concentração acionária e da estrutura do CA sobre o nível de remuneração executiva. Seus achados trazem implicações para a compreensão dos principais conflitos de agência e para a governança no mercado de capitais brasileiro.

A evidência empírica atestada de que empresas brasileiras são caracterizadas pela alta concentração acionária nas mãos de poucos acionistas é relevante, pois esta leva a efeitos específicos sobre políticas estratégicas da empresa relacionadas a níveis de remuneração executiva.

De fato, em linha com estudos sobre o tema, a primeira contribuição desta pesquisa está na confirmação de que a concentração acionária tem influência negativa relevante sobre o nível de remuneração da alta gestão, evidenciando que o monitoramento exercido pelo controlador sobre a gestão parece ser mais atuante na mitigação dos clássicos conflitos agenteprincipal do que a remuneração paga à diretoria. No entanto, o exercício de monitoramento direto pelo controlador substitui função típica do CA, cuja atuação torna-se limitada.
Esse nível mais baixo de remuneração que o controlador está disposto a pagar a seus executivos pode ser justificado pela busca de incentivos para que aqueles possam se favorecer com benefícios privados de controle, o que, de outra maneira, repercute, sob a forma de expropriação de minoritários, no agravamento dos conflitos principal-principal.

Por outro lado, na análise da influência da estrutura do CA sobre a remuneração executiva, tem-se, como segunda contribuição da pesquisa, que a Abordagem do Poder Gerencial parece não se adequar ao Brasil, porquanto, embora influencie o CA, a diretoria não detém poder para extrair níveis de remuneração mais elevados, o que pode ser explicado, num contexto institucional de alta concentração de propriedade, pelo predomínio do conflito principal-principal no âmbito da firma brasileira.

Vale destacar, ainda, que a estrutura do CA, ao alterar a sensibilidade da remuneração à concentração acionária, reforça o argumento de que a baixa qualidade de composição do CA está associada a ambientes de alta concentração de propriedade. Tal constatação, terceira contribuição do estudo, evidencia que o controlador, ao exercer influência sobre o nível de remuneração executiva, utiliza o CA tanto para expropriar minoritários ao extrair benefícios privados de controle quanto para substituí-lo na função de monitoramento da gestão. Assim, espera-se que esses achados possam contribuir para as decisões de melhoria de processos de governança nas firmas.

Embora o estudo apresente limitações, destacando-se o exíguo período de análise e tamanho reduzido da amostra, acredita-se que ele contribui para enriquecer a compreensão e debate sobre a dinâmica dos principais conflitos de agência presentes no mercado acionário brasileiro a partir da interação entre concentração acionária, estrutura do CA e remuneração executiva, ainda pouco explorada, oferecendo elementos para uma discussão mais aprofundada sobre seus possíveis impactos no mundo corporativo.

Destarte, sugere-se que pesquisas futuras venham ampliar o período e amostra, expandindo-a para empresas de outras economias, incorporando aspectos institucionais, com aplicação de métodos estatísticos mais robustos, além de considerar outros mecanismos de governança.

\section{REFERÊNCIAS}

Abascal, R., \& González, F. (2019). Shareholder protection and bank executive compensation after the global financial crisis. Journal of Financial Stability, 40, 15-37. doi:10.1016/j.jfs.2018.11.004 
Almazan, A., Hartzell, J. C., \& Starks, L. T. (2005). Active institutional shareholders and costs of monitoring: Evidence from executive compensation. Financial Management, 34(4), 5-34. doi:10.1111/ j.1755-053X.2005.tboo116.x

Anjos, L. C. M., Tavares, M. F. M., Monte, P. A., \& Lustosa, P. R. B. (2015). Relações entre controle acionário e remuneração de executivos. Enfoque, 34(1), 45-56. doi:10.4025/enfoque.v34i1.22493

Bebchuk, L. A., \& Fried, J. M. (2003). Executive compensation as an agency problem. Journal of Economic Perspectives, 17(3), 71-92. doi:10.1257/089533003769204362

Bebchuk, L. A., \& Fried, J. M. (2004). Pay without performance: The unfulfilled promise of executive compensation. Cambridge, USA: Harvard University Press.

Berle, A. A., \& Means, G. C. (1932). The modern corporation and private property. New York, USA: Transaction Publishers.

Bertucci, J. L. O., Bernardes, P., \& Brandão, M. M. (2006). Políticas e práticas de governança corporativa em empresas brasileiras de capital aberto. Revista de Administração, 41(2), 183-196. doi:10.1590/ Soo80-21072006000200006

Bettis, J. C., Bizjak, J., Coles, J., \& Kalpathy, S. (2018). Performancevesting provisions in executive compensation. Journal of Accounting and Economics, 66(1), 194-221. doi: 0.1016/j.jacceco.2018.05.001

Bozec, Y., \& Bozec, R. (2007). Ownership concentration and corporate governance practices: Substitution or expropriation effects? Canadian Journal of Administrative Sciences, 24(3), 185-195. doi: $10.1002 /$ cjas.23

Brandão, I. F., \& Crisóstomo, V. L. (2015). Concentração de propriedade e qualidade da governança da empresa brasileira. Revista Brasileira de Finanças, 13(3), 438-472. doi: 10.1266o/rbfin.v13n3.2015.45739

Brandão, I. F., Vasconcelos, A. C., Luca, M. M. M. De, \& Crisóstomo, V. L. (2019). Composição do conselho de administração e sensibilidade da remuneração executiva ao desempenho de mercado. Revista Contabilidade \& Finanças, 30(79), 28-41. doi: 10.1590/1808$057 \times 201806610$

Bugeja, M., Rosa, R. S., Duong, L., \& Izan, H.Y. (2012). CEO compensation from M\&As in Australia. Journal of Business Finance \& Accounting, 39(9-10), 1298-1329. doi: 10.1111/j.1468-5957.2012.02299.x

Cao, J., Pan, X., Tian, G. (2011). Disproportional ownership structure and pay-performance relationship: Evidence from China's listed firms. Journal of Corporate Finance, 17(3), 541-554. doi: 10.1016/j. jcorpfin.2011.02.006

Chen, Z., \& Keefe, M. O. C. (2018). Board of director compensation in China: To pay or not to pay? How much to pay? Emerging Markets Review, 37(C), 66-82. doi: 10.1016/j.ememar.2018.05.003

Cheung, Y. L., Stouraitis, A, \& Wong, A. W. S. (2005). Ownership concentration and executive compensation in closely held firms: Evidence from Hong Kong. Journal of Empirical Finance, 12, 511-532. doi:10.1016/j.jempfin.2004.10.001

Chhaochharia, V., \& Grinstein, Y. (2009). CEO compensation and board structure. The Journal of Finance, 64(1), 231-261. doi: 10.1111/j.15406261.2008.01433.x

Choe, C., Tian, G. Y., \& Yin, X. (2014). CEO power and the structure of CEO pay. International Review of Financial Analysis, 35(5), 237-248. doi: 10.1016/j.irfa.2014.10.004
Claessens, S., \& Yurtoglu, B. B. (2013). Corporate governance in emerging markets: A survey. Emerging Markets Review, 15(2), 1-33. doi: 10.1016/j.ememar.2012.03.002

Conyon, M. J., \& Peck, S. I. (1997). Board control, remuneration committees, and top management compensation. Academy of Management Journal, 41(2), 146-157. doi: 10.2307/257099

Core, J. E., Holthausen, R. W., \& Larcker, F. D. (1999). Corporate governance, chief executive compensation, and firm performance. Journal of Financial Economics, 51(3), 371-406. doi: 10.1016/S0304405X(98)00058-0

Correia, L. F., Amaral, H. F., \& Louvet, P. (2014). Remuneração, composição do conselho de administração e estrutura de propriedade: Evidências empíricas do mercado acionário brasileiro. Advances in Scientific and Applied Accounting, 7(1), 2-37.doi: 10.14392/ASAA.2014070101

Crisóstomo, V. L., Brandão, I. F., \& Lopéz-Iturriaga, F. J. (2020). Large shareholders'power and the quality of corporate governance: An analysis of Brazilian firms. Research in International Business and Finance, 51(C), 1-15. doi:10.1016/j.ribaf.2019.101076

Croci, E., Gonenc, H., \& Ozkan, N. (2012). CEO compensation, family control, and institutional investors in Continental Europe. Journal of Banking \& Finance, 36(12), 3318-3335. doi:10.1016/j. jbankfin.2012.07.017

Dutra, M. G. R., \& Saito, L. (2002). Conselhos de administração: Análise de sua composição em um conjunto de companhias abertas brasileiras. Revista de Administração Contemporânea, 6(2), 9-27. doi:10.1590/S1415-65552002000200003.

Dyck, A., \& Zingales, L. (2004). Private benefits of control: An international comparison. The Journal of Finance, 59(2), 537-600. doi:10.1111/j.1540-6261.2004.00642.x

Ermel, M. D. A., \& Monte, P. A. (2016). Controle acionário, remuneração de executivos e desempenho empresarial: Evidências para o mercado brasileiro. FGV/EAESP Working Papers Series, 6(1), 1-32. doi:10.26512/2015.06.D.19144

Fávero, L. P., Belfiore, P. P., Silva, F. L. da, \& Chan, B. L. (2009). Análise de dados: Modelagem multivariada para tomada de decisões. Rio de Janeiro, RJ: Elsevier.

Finkelstein, S. (1992). Power in top management teams: Dimensions, measurement, and validation. Academy of Management Journal, 35(3), 505-538. doi:10.2307/256485

Firth, M., Fung, P. M. Y., \& Rui, O. M. (2007). How ownership and corporate governance influence chief executive pay in China's listed firms. Journal of Business Research, 6o(7), 776-785. doi:10.1016/j. jbusres.2007.01.014

Grabke-Rundell, A., \& Gomez-Mejia, L. R. (2002). Power as a determinant of executive compensation. Human Resource Management Review, 12(1), 3-23. doi:10.1016/S1053-4822(01)00038-9

Hartzell, J. C., \& Starks, L. T. (2003). Institutional investors and executive compensation. Journal of Finance, 58(6), 2351-2374. doi:10.1046/ j.1540-6261.2003.00608.x

Hassen, R. B., Ouakdi, J. E., \& Omri, A. (2015). Executive compensation and ownership structure. The Journal of Applied Business Research, 31(2), 593-609. doi:10.19030/jabr.v31i2.9156

Instituto Brasileiro de Governança Corporativa. (2015). Código brasileiro de governança corporativa: Companhias abertas. São Paulo, SP: IBGC. 
Jensen, M. C. (1993). The modern industrial revolution, exit, and the failure of internal control systems. The Journal of Finance, 48(3), 831880. doi: 10.1111/j.1540-6261.1993.tbo4022.x

Jensen, M. C, \& Meckling, W. H. (1976). Theory of the firm: Managerial behavior, agency costs and ownership structure. Journal of Financial Economics, 3(4), 305-360. doi: 10.1016/0304-405X(76)90026-X

Jiang, H., Habib, A., \& Smallman, C. (2009). The effect of ownership concentration on CEO compensation-firm performance relationship in New Zealand. Pacific Accounting Review, 21(2), 104-131. doi:0.1108/01140580911002053

John, K., Mehran, H., \& Qian, Y. M. (2010). Outside monitoring and CEO compensation in the banking industry. Journal of Corporate Finance, 16(4), 383-399. doi:10.1016/j.jcorpfin.2010.01.001

Lee, S. P., \& Chen, H. J. (2011). Corporate governance and firm value as determinants of CEO compensation in Taiwan. Management Research Review, 34(3), 252-265. doi:10.1108/01409171111116286

Lin, B. X., \& Lu, R. (2009). Managerial power, compensation gap and firm performance- evidence from Chinese public listed companies. Global Finance Journal, 20(2), 153-164. doi:10.1016/j.gfj.2008.12.002

Luo, Y. (2015). CEO power, ownership structure and pay performance in Chinese banking. Journal of Economics and Busines, 82(C), 3-16. doi:10.1016/j.jeconbus.2015.04.003

Mehran, H. (1995). Executive compensation structure, ownership, and firm performance. Journal of Financial Economics, 38(2), 163-184. doi:10.1016/0304-405X(94)00809-F

Newman, H. A., \& Mozes, A. H. (1999). Does the composition of the compensation committee influence CEO compensation practices? Financial Management, 28(3), 41-53. doi: 10.2307/3666182

Ozdemir, O., \& Upneja, A. (2012). Board structure and CEO compensation: Evidence from U.S. lodging industry. International Journal of Hospitality Management, 31(3), 856-863. doi: 10.1016/j. ijhm.2011.10.004
Pinto, M. B., \& Leal, R. P. C. (2013). Ownership concentration, top management and board compensation. Revista de Administração Contemporânea, 17(3), 304-324. doi: 10.1590/S1415-65552013000300004

Porta, R., Lopez-de-Silanes, F., \& Shleifer, A. (1999). Corporate ownership around the world. The Journal of Finance, 54(2), 471-517. doi:10.1111/0022-1082.00115

Porta, R., López-de-Silanes, F., Shleifer, A., \& Vishny, R. W. (2000). Investor protection and corporate governance. Journal of Financial Economics, 58(1-2), 3-27. doi:10.1016/S0304-405X(00)000659

Schiehll, E., \& Santos, I. O. (2004). Ownership structure and composition of boards of directors: Evidence on Brazilian publicly traded companies. Revista de Administração, 39(4), 373-384. Recuperado de http://200.232.30.99/busca/artigo.asp?num_artigo=1138

Shleifer, A., \& Vishny, R. (1997). A survey of corporate governance. Journal of Finance, 52(2), 737-783. doi:10.1111/j.1540-6261.1997. tbo4820.x

Silveira, A. D. M., Leal, R. P. C., Carvalhal-da-Silva, A. L., \& Barros, L. A. B. C. (2010). Endogeneity of Brazilian corporate governance quality determinants. Corporate Governance, 10(2), 191-202. doi:10.1108/14720701011035701

Theiss, V., \& Beuren, I. M. (2017). Estrutura de propriedade e remuneração dos executivos. Revista de Administração da UFSM, 10(3), 533-550. doi:10.5902/1983465913062

Young, M. N., Peng, M. W., Ahlstrom, D., Bruton, G. D., \& Jiang, Y. (2008). Corporate governance in emerging economies: A review of the principal-principal perspective. Journal of Management Studies, 45(1), 196-220. doi:10.1111/j.1467-6486.2007.00752.x

\section{CONTRIBUICAO DOS AUTORES}

Os autores desenvolveram juntos todo o trabalho deste artigo, desde a sua conceitualização e abordagem teóricametodológica, revisão teórica (levantamento de literatura), coleta e análise de dados, até a redação e revisão final do texto. 